\title{
SYMPLECTIC DIFFERENCE SYSTEMS: OSCILLATION THEORY AND HYPERBOLIC PRÜFER TRANSFORMATION
}

\author{
ONDŘEJ DOŠLÝ
}

Received 8 October 2002

We present basic methods of oscillation theory of symplectic difference systems (SDSs). A particular attention is devoted to the variational principle and to the transformation method. Hyperbolic Prüfer transformation for SDSs is established.

\section{Introduction}

In this paper, we deal with oscillatory properties and transformations of symplectic difference systems (SDSs)

$$
z_{k+1}=\mathscr{I}_{k} z_{k}, \quad z_{k}=\left(\begin{array}{l}
x_{k} \\
u_{k}
\end{array}\right), \mathscr{I}_{k}=\left(\begin{array}{ll}
\mathscr{A}_{k} & \mathscr{B}_{k} \\
\mathscr{C}_{k} & \mathscr{D}_{k}
\end{array}\right),
$$

where $x, u \in \mathbb{R}^{n}, \mathscr{A}, \mathscr{B}, \mathscr{C}, \mathscr{D} \in \mathbb{R}^{n \times n}$, and the matrix $\mathscr{Y}$ is supposed to be symplectic, that is,

$$
\mathscr{S}_{k}^{T} \mathscr{G} \mathscr{Y}_{k}=\mathscr{F}, \quad \mathscr{F}=\left(\begin{array}{cc}
0 & I \\
-I & 0
\end{array}\right) .
$$

The last identity translates in terms of the block entries $\mathscr{A}, \mathscr{B}, \mathscr{C}$, and $\mathscr{D}$ as

$$
\mathscr{A}^{T} \mathscr{C}=\mathscr{C}^{T} \mathscr{A}, \quad \mathscr{B}^{T} \mathscr{D}=\mathscr{D}^{T} \mathscr{B}, \quad \mathscr{A}^{T} \mathscr{D}-\mathscr{C}^{T} \mathscr{B}=I .
$$

If $Z=\left(\begin{array}{l}X \\ U\end{array}\right), \tilde{Z}=\left(\begin{array}{c}\tilde{X} \\ \tilde{U}\end{array}\right)$ are $2 n \times n$ matrix solutions of $(1.1)$ and $\mathscr{E}=(Z \tilde{Z})=\left(\begin{array}{cc}X & \tilde{X} \\ U & \tilde{U}\end{array}\right)$, then using (1.2), we have

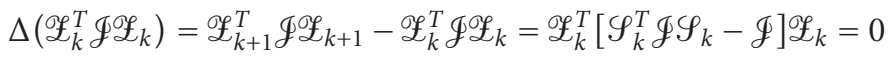

which means that $\mathscr{L}_{k}$ are symplectic whenever this property is satisfied at one index, say $k=0$. Consequently, (1.1) defines the discrete symplectic flow and this fact, together with (1.2), is the justification for the terminology symplectic difference system. 
SDSs cover, as particular cases, a large variety of difference equations and systems, among them the Sturm-Liouville second-order difference equation

$$
\Delta\left(r_{k} \Delta x_{k}\right)+p_{k} x_{k+1}=0, \quad r_{k} \neq 0, \Delta x_{k}=x_{k+1}-x_{k},
$$

the higher-order selfadjoint difference equation

$$
\sum_{\nu=0}^{n}(-1)^{\nu} \Delta^{\nu}\left(r_{k}^{[\nu]} \Delta^{\nu} y_{k+n-\nu}\right)=0, \quad r_{k}^{[n]} \neq 0, \Delta^{\nu}=\Delta\left(\Delta^{\nu-1}\right),
$$

and the linear Hamiltonian difference system

$$
\Delta x_{k}=A_{k} x_{k+1}+B_{k} u_{k}, \quad \Delta u_{k}=C_{k} x_{k+1}-A_{k}^{T} u_{k}
$$

with $A, B, C \in \mathbb{R}^{n \times n}, B$ and $C$ symmetric (i.e., $B=B^{T}, C=C^{T}$ ), and $I-A$ invertible.

Our paper is organized as follows. In the remaining part of this section we recall, for the sake of later comparison, basic oscillatory properties of the Sturm-Liouville equation (1.5). Section 2 contains the so-called Roundabout theorem for (1.1) which forms the basis for the investigation of oscillatory properties of these systems. We also mention some results concerning transformations of (1.1). Section 3 is devoted to the illustration of the methods of oscillation theory of (1.1) and Section 4 contains a new result, the socalled discrete hyperbolic Prüfer transformation. We also formulate some open problems associated with this type of transformation.

Now, we recall basic facts of the oscillation theory of (1.5) as can be found, for example, in $[1,2,11,14]$. We substitute $u=r \Delta x$ in (1.5). Then this equation can be written as a $2 \times 2$ Hamiltonian system $(1.7)$

$$
\Delta\left(\begin{array}{l}
x_{k} \\
u_{k}
\end{array}\right)=\left(\begin{array}{cc}
0 & \frac{1}{r_{k}} \\
-p_{k} & 0
\end{array}\right)\left(\begin{array}{c}
x_{k+1} \\
u_{k}
\end{array}\right)
$$

and expanding the forward differences as a $2 \times 2$ symplectic system

$$
\left(\begin{array}{l}
x_{k+1} \\
u_{k+1}
\end{array}\right)=\left(\begin{array}{cc}
1 & \frac{1}{r_{k}} \\
-\frac{p_{k}}{r_{k}} & 1-\frac{p_{k}}{r_{k}}
\end{array}\right)\left(\begin{array}{l}
x_{k} \\
u_{k}
\end{array}\right) .
$$

We say that an interval $(m, m+1]$ contains a focal point (an alternative terminology is generalized zero, see [13]) of a solution $x$ of (1.5) if $x_{m} \neq 0$ and $r_{m} x_{m} x_{m+1} \leq 0$. Equation (1.5) is said to be disconjugate in the discrete interval $[0, N]$ if the solution $\tilde{x}$ given by the initial condition $\tilde{x}_{0}=0, \tilde{x}_{1}=1 / r_{0}$ has no focal point in $(0, N+1]$. This equation is said to be nonoscillatory if there exists $n \in \mathbb{N}$ such that (1.5) is disconjugate on $[n, m]$ for every $m>n$, and it is said to be oscillatory in the opposite case.

The next statement, usually referred to as the Roundabout theorem, shows that the discrete quadratic functional and the discrete Riccati equation play the same role in the oscillation theory of (1.5) as their continuous counterparts in the oscillation theory of 
the Sturm-Liouville differential equation

$$
\left(r(t) x^{\prime}\right)^{\prime}+p(t) x=0
$$

Proposition 1.1. The following statements are equivalent:

(i) equation (1.5) is disconjugate on the interval $[0, N]$,

(ii) there exists a solution $x$ of (1.5) having no focal point in $[0, N+1]$,

(iii) there exists a solution $w$ of the Riccati equation (related to (1.5) by the substitution $w=r \Delta x / x)$

$$
\Delta w_{k}+p_{k}+\frac{w_{k}^{2}}{w_{k}+r_{k}}=0
$$

which is defined for $k \in[0, N+1]$ and satisfies $r_{k}+w_{k}>0$ for $k \in[0, N]$,

(iv) the quadratic functional

$$
\sum_{k=0}^{N}\left\{r_{k}\left(\Delta y_{k}\right)^{2}-p_{k} y_{k+1}^{2}\right\}>0
$$

for every nontrivial $y=\left\{y_{k}\right\}_{k=0}^{N+1}$ with $y_{0}=0=y_{N+1}$.

Note that the previous proposition actually shows that the Sturmian separation and comparison theory extend verbatim to (1.5), using the same argument as in the case of the differential equation (1.10).

\section{Oscillation theory of SDSs}

First, we turn our attention to Hamiltonian difference systems (1.7). Oscillation theory of these systems attracted considerable attention in late eighties and early nineties of the last century (see $[8,12]$ and the references given therein). Note that in both of these papers, it is assumed that the matrix $B$ is positive definite and hence nonsingular. However, such Hamiltonian systems do not cover several important equations, for example, (1.6), in which case the matrix $B=\operatorname{diag}\left\{0, \ldots, 0,1 / r_{n}^{[n]}\right\}$ in the Hamiltonian system corresponding to this equation. This difficulty was removed in the pioneering paper of Bohner [3], where the concept of the focal point of a matrix solution of (1.7) with $B$ possibly singular was introduced. Later, this concept was extended to system (1.1) in [5] and reads as follows. We say that a conjoined basis $Z=\left(\begin{array}{l}X \\ U\end{array}\right)$ of (1.1) (i.e., a $2 n \times n$ matrix solution such that $X^{T} U$ is symmetric and $\left.\operatorname{rank}\left(\begin{array}{l}X \\ U\end{array}\right) \equiv n\right)$ has a focal point in an interval $(m, m+1], m \in \mathbb{Z}$, if $\operatorname{Ker} X_{m+1} \nsubseteq \operatorname{Ker} X_{m}$ or “ $\subseteq$ ” holds, but $P_{m}:=X_{m} X_{m+1}^{\dagger} \mathscr{B}_{m} ¥ 0$, here Ker, ${ }^{\dagger}$, and $\geq$ mean the kernel, the generalized inverse, and nonnegative definiteness of a symmetric matrix, respectively. Note that if the inclusion " $\subseteq$ " holds, then the matrix $P_{m}$ is really symmetric (see [5]). System $(1.1)$ is said to be disconjugate on $[0, N]$ if the solution $Z=\left(\begin{array}{l}X \\ U\end{array}\right)$ given by the initial condition $X_{0}=0, U_{0}=I$ has no focal point in $(0, N+1]$. Oscillation and nonoscillation of (1.1) are defined via disconjugacy in the same way as for (1.5).

The following statement shows that, similar to the scalar case, certain discrete quadratic functional and Riccati-type difference equation play a crucial role in the oscillation 
theory of (1.1). This statement is proved in [5] and we present it here in a slightly modified form.

Proposition 2.1. The following statements are equivalent:

(i) system (1.1) is disconjugate in the interval $[0, N]$,

(ii) there exists a conjoined basis $Z=\left(\begin{array}{l}X \\ U\end{array}\right)$ without any focal point in $[0, N+1]$ and with $X_{k}$ nonsingular in this interval,

(iii) there exists a symmetric solution $Q$ of the Riccati matrix difference equation

$$
Q_{k+1}=\left(\mathscr{C}_{k}+\mathscr{D}_{k} Q_{k}\right)\left(\mathscr{A}_{k}+\mathscr{B}_{k} Q_{k}\right)^{-1}
$$

which is defined for $k \in[0, N+1]$ and the matrix $P_{k}:=\mathscr{B}_{k}^{T}\left(\mathscr{D}_{k}-Q_{k+1} \mathscr{B}_{k}\right)$ is non negative definite for $k \in[0, N]$,

(iv) let $\mathscr{K}=\left(\begin{array}{ll}0 & 0 \\ I & 0\end{array}\right)$. The quadratic functional corresponding to (1.1)

$$
\begin{aligned}
\mathscr{F}(z) & =\sum_{k=0}^{N} z_{k}^{T}\left\{\mathscr{S}_{k}^{T} \mathscr{H}-\mathscr{K}\right\} z_{k} \\
& =\sum_{k=0}^{N}\left\{x_{k}^{T} \mathscr{C}_{k}^{T} \mathscr{A}_{k} x_{k}+2 x_{k}^{T} \mathscr{C}_{k}^{T} \mathscr{B}_{k} u_{k}+u_{k}^{T} \mathscr{D}_{k}^{T} \mathscr{B}_{k} u_{k}\right\}
\end{aligned}
$$

is positive for every $z=\left\{z_{k}\right\}_{k=0}^{N+1}$ satisfying $\mathscr{K} z_{k+1}=\mathscr{K} \mathscr{T}_{k} z_{k}, \mathscr{K} z_{0}=0=\mathscr{K} z_{N+1}$, and $\mathscr{K} z \not \equiv 0$, that is, if we write $z=\left(\begin{array}{l}x \\ u\end{array}\right)$, then $\mathscr{F}(x, u)>0$ for every $x$, $u$ satisfying $x_{k+1}=$ $\mathscr{A}_{k} x_{k}+\mathscr{B}_{k} u_{k}, x_{0}=0=x_{N+1}, x \neq 0$.

It is not difficult to verify that if (1.1) is the rewritten equation (1.5), that is, $\mathscr{A}=1, \mathscr{B}=$ $1 / r, \mathscr{C}=-p$, and $\mathscr{D}=1-p / r$, then the objects appearing in the previous proposition reduce to their scalar counterparts mentioned in Proposition 1.1.

We finish this section with a short description of the transformation theory of (1.1). Let $\mathscr{R}_{k}$ be symplectic matrices and consider the transformation of (1.1)

$$
z_{k}=\mathscr{R}_{k} \tilde{z}_{k}
$$

This transformation transforms (1.1) into the system

$$
\tilde{z}_{k+1}=\tilde{\mathscr{S}}_{k} \tilde{z}_{k}, \quad \tilde{\mathscr{S}}_{k}=\mathscr{R}_{k+1}^{-1} \mathscr{S}_{k} \mathscr{R}_{k}
$$

which is again a symplectic system as can be verified by a direct computation. The case when $\mathscr{R}$ is of the form

$$
\mathscr{R}_{k}=\left(\begin{array}{cc}
H_{k} & 0 \\
G_{k} & H_{k}^{T-1}
\end{array}\right)
$$

is of particular importance in oscillation theory of (1.1). In this case, transformation (2.3) preserves the oscillatory nature of transformed systems (see [5]) and if we write 
$\tilde{\mathscr{S}}=\left(\begin{array}{cc}\tilde{\mathscr{A}} & \tilde{\mathscr{P}} \\ \tilde{\mathscr{C}} & \tilde{\mathscr{D}}\end{array}\right)$, then we have

$$
\begin{aligned}
& \tilde{\mathscr{A}}_{k}=H_{k+1}^{-1}\left(\mathscr{A}_{k} H_{k}+\mathscr{B}_{k} G_{k}\right), \quad \tilde{\mathscr{B}}_{k}=H_{k+1}^{-1} \mathscr{B}_{k} H_{k}^{T-1}, \\
& \tilde{\mathscr{C}}_{k}=H_{k+1}^{T}\left(\mathscr{C}_{k} H_{k}+\mathscr{D}_{k} G_{k}\right)-G_{k+1}^{T}\left(\mathscr{A}_{k} H_{k}+\mathscr{B}_{k} G_{k}\right), \\
& \tilde{\mathscr{D}}_{k}=H_{k+1}^{T} \mathscr{D}_{k} H_{k}^{T-1}-G_{k+1}^{T} \mathscr{B}_{k} H_{k}^{T-1} .
\end{aligned}
$$

Consequently, transformation (2.3), with $\mathscr{R}$ of the form (2.5), is a useful tool in the oscillation theory of (1.1); this system is transformed into an "easier" system and the results obtained for this "easier" system are then transformed back to the original system. For some oscillation results obtained in this way, we refer to $[6,9]$.

\section{Oscillation theory of SDSs}

In addition to the transformation method mentioned in Section 2, the Roundabout theorem (Proposition 2.1) suggests two other methods of the oscillation theory of these systems. The first one, the so-called Riccati technique, consists in the equivalence (i) $\Longleftrightarrow$ (iii). The oscillation results for (1.7) with $B$ positive definite, mentioned at the beginning of Section 2, were proved just using this method. However, as we have already mentioned, this method does not extend directly to a Hamiltonian system with $B$ singular or to general SDSs. It is an open problem (which is the subject of the present investigation) how to modify this method in order to be applicable also in the more general situation.

The second principal method of the oscillation theory of (1.1), the so-called variational principle, is based on the equivalence of disconjugacy and positivity of quadratic functional (2.2), which is the equivalence (i) $\Leftrightarrow$ (iv) in Proposition 2.1. Unlike the Riccati technique, this method extends to general SDSs almost without problems and the illustration of this extension is the main part of this section.

The discrete version of the classical Leighton-Wintner criterion for the Sturm-Liouville differential equation (1.10) states that the Sturm-Liouville difference equation (1.5) with $r_{k}>0$ is oscillatory provided

$$
\sum \frac{1}{r_{k}}=\infty, \quad \sum p_{k}=\infty .
$$

In this criterion, equation (1.5) is essentially viewed as a perturbation of the one-term (nonoscillatory) equation

$$
\Delta\left(r_{k} \Delta x_{k}\right)=0
$$

According to the equivalence of oscillation of (1.5) and the existence of a sequence (with zero boundary values) for which the associated quadratic functional (1.12) is nonpositive, for the oscillation of the "perturbed" equation (1.5), the sequence $p_{k}$ must be, in a certain sense, sufficiently positive. The second condition in (3.1) is just a quantitative characterization of the "sufficient positivity" of $p_{k}$. 
Now, we show how this criterion extends to (1.1). Let $\tilde{\mathscr{C}}_{k}$ be a sequence of symmetric nonpositive definite $n \times n$ matrices and consider the system

$$
z_{k+1}=\left(\mathscr{S}_{k}+\tilde{\mathscr{S}}_{k}\right) z_{k}, \quad \tilde{\mathscr{S}}_{k}=\left(\begin{array}{cc}
0 & 0 \\
\tilde{\mathscr{C}}_{k} \mathscr{A}_{k} & \tilde{\mathscr{C}}_{k} \mathscr{B}_{k}
\end{array}\right),
$$

as a perturbation of (1.1). The quadratic functional corresponding to (3.3) has the same class of admissible pairs $x, u$, as the functional corresponding to (1.1), and takes the form

$$
\begin{aligned}
& \tilde{\mathscr{F}}_{k}(x, u)=\sum_{k=0}^{N}\left\{x_{k}^{T}\left(\mathscr{C}_{k}+\tilde{\mathscr{C}}_{k} \mathscr{A}_{k}\right)^{T} \mathscr{A}_{k} x_{k}+2 x_{k}^{T}\left(\mathscr{C}_{k}+\tilde{\mathscr{C}}_{k} \mathscr{A}_{k}\right)^{T} \mathscr{B}_{k} u_{k}\right.
\end{aligned}
$$

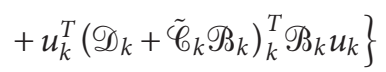

$$
\begin{aligned}
& =\sum_{k=0}^{N}\left\{x_{k}^{T} \mathscr{C}_{k}^{T} \mathscr{A}_{k} x_{k}+2 x_{k}^{T} \mathscr{C}_{k}^{T} \mathscr{B}_{k} u_{k}+u_{k}^{T} \mathscr{D}_{k}^{T} \mathscr{B}_{k} u_{k}\right\}+\sum_{k=0}^{N} x_{k+1}^{T} \tilde{\mathscr{C}}_{k} x_{k+1} \text {. }
\end{aligned}
$$

In our extension of the Leighton-Wintner-type criterion to (1.1), we will need two additional concepts of the oscillation theory of these systems. System (1.1) is said to be eventually controllable if there exists $N \in \mathbb{N}$ such that the trivial solution $z=\left(\begin{array}{l}x \\ u\end{array}\right) \equiv\left(\begin{array}{l}0 \\ 0\end{array}\right)$ is the only solution for which $x_{k}=0$ for $k \geq N$. A conjoined basis $\tilde{Z}=\left(\begin{array}{l}\tilde{X} \\ \tilde{U}\end{array}\right)$ is said to be the recessive solution of (1.1) at $\infty$ if there exists $N \in \mathbb{N}$ such that $\tilde{X}_{k}$ is nonsingular for $k \geq N$ and

$$
\lim _{k \rightarrow \infty}\left(\sum_{j=N}^{k} \tilde{X}_{j+1}^{-1} \mathscr{\Re}_{j} \tilde{X}_{j}^{T-1}\right)^{-1}=0 .
$$

Note that the principal solution at $\infty$ exists and it is unique (up to the right multiplication by a nonsingular constant matrix) whenever (1.1) is nonoscillatory and eventually controllable (see [5]).

THeorem 3.1. Suppose that (1.1) is nonoscillatory, eventually controllable and let $\tilde{Z}=\left(\begin{array}{c}\tilde{X} \\ \tilde{U}\end{array}\right)$ be the principal solution at $\infty$ of this system. If there exists a vector $v \in \mathbb{R}^{n}$ such that

$$
\sum^{\infty} v^{T} \tilde{X}_{k+1}^{T} \tilde{\mathscr{C}}_{k} \tilde{X}_{k+1} v=-\infty
$$

then (3.3) is oscillatory.

We skip the proof of this statement which is based on a rather complicated construction of an admissible pair $x, u$ for which $\tilde{\mathscr{F}}(x, u)<0$ (for details, see [4]). We concentrate our attention on showing that the previous theorem is really an extension of the Leighton-Wintner criterion (3.1). Equation (3.2) can be written as the $2 \times 2$ symplectic system

$$
\left(\begin{array}{l}
x \\
u
\end{array}\right)_{k+1}=\left(\begin{array}{cc}
1 & \frac{1}{r_{k}} \\
0 & 1
\end{array}\right)\left(\begin{array}{l}
x \\
u
\end{array}\right)_{k}
$$


which plays the role of system (1.1). The perturbation quantity is $\tilde{\mathscr{C}}=-p$, that is, $\tilde{\mathscr{S}}=$ $\left(\begin{array}{cc}0 & 0 \\ -p & -p / r\end{array}\right)$, and hence the symplectic system (1.9) (which is the rewritten equation (1.5)) plays the role of (3.3). Now, the first condition in (3.1) is

$$
\sum \frac{1}{r_{k}}=\infty \Longleftrightarrow \lim _{k \rightarrow \infty}\left(\sum \frac{1}{r_{j}}\right)^{-1}=0
$$

which means that $\tilde{x}_{k} \equiv 1$ is the principal solution at $\infty$ of $(3.2)$. Since $\tilde{\mathscr{C}}=-p$, clearly (3.6) with $v=1, n=1$, and $\tilde{X}=\tilde{x}=1$ is equivalent to the second condition in (3.1). Hence, the Leighton-Wintner oscillation criterion (3.1) is really a consequence of Theorem 3.1.

\section{Hyperbolic Prüfer transformation}

The classical Prüfer transformation (established by Prüfer in [15]) is a useful tool in the qualitative theory of the second-order Sturm-Liouville differential equation

$$
\left(r(t) x^{\prime}\right)^{\prime}+p(t) x=0
$$

where $r$ and $p$ are continuous functions with $r(t)>0$. By this transformation, any non trivial solution $x$ of (4.1) and its quasiderivative $r x^{\prime}$ can be expressed in the form

$$
x(t)=\rho(t) \sin \varphi(t), \quad r(t) x^{\prime}(t)=\rho(t) \cos \varphi(t),
$$

where $\rho$ and $\varphi$ satisfy the first-order system

$$
\varphi^{\prime}=p(t) \sin ^{2} \varphi+\frac{1}{r(t)} \cos ^{2} \varphi, \quad \rho^{\prime}=\frac{1}{2} \sin 2 \varphi(t)\left(\frac{1}{r(t)}-p(t)\right) \rho .
$$

Since 1926, when the original paper of Prüfer appeared, the Prüfer transformation has been extended in various directions (see [7] and the references given therein). Here, we present another extension: the so-called hyperbolic discrete Prüfer transformation which is based on the following idea. If the Sturm-Liouville equation (4.1) possesses a solution $x$ such that $\left(r(t) x^{\prime}(t)\right)^{2}-x^{2}(t)>0$ in some interval $I \subset \mathbb{R}$, then the solution $x$ and its quasiderivative $r x^{\prime}$ can be expressed via the hyperbolic sine and cosine functions in the form

$$
x(t)=\rho(t) \sinh \varphi(t), \quad r(t) x^{\prime}(t)=\rho(t) \cosh \varphi(t)
$$

in this interval, where the functions $\rho$ and $\varphi$ satisfy a first-order system similar to (4.3). The crucial role in our extension of this transformation is played by the so-called hyperbolic symplectic system, which is the SDS of the form

$$
x_{k+1}=\mathscr{P}_{k} x_{k}+\mathscr{2}_{k} u_{k}, \quad u_{k+1}=\mathscr{2}_{k} x_{k}+\mathscr{P}_{k} u_{k},
$$

that is, the $n \times n$ matrices $\mathscr{P}$ and $\mathscr{Q}$ satisfy

$$
\mathscr{P}^{T} \mathscr{P}-\mathscr{2}^{T} \mathscr{Q}=I, \quad \mathscr{P}^{T} \mathscr{Q}-\mathscr{2}^{T} \mathscr{P}=0 .
$$


Note that the terminology hyperbolic symplectic system is motivated by the fact that in the scalar case $n=1$, solutions of (4.5) are, in case $\mathscr{P}_{k}>0$, of the form

$$
x_{k}=\sinh \left(\sum \sum^{k-1} \varphi_{j}\right), \quad u_{k}=\cosh \left(\sum-1 \varphi_{j}\right)
$$

where $\varphi_{k}$ is a sequence given by $\cosh \varphi_{k}=\mathscr{P}_{k}, \sinh \varphi_{k}=\mathscr{2}_{k}$. For basic properties of solutions of hyperbolic symplectic systems, we refer to [10].

Theorem 4.1. Suppose that (1.1) possesses a conjoined basis $(\underset{U}{X})$ such that $U_{k}^{T} U_{k}-X_{k}^{T} X_{k}$ is positive definite for $k$ in some discrete interval $[m, n], m, n \in \mathbb{N}$. Then there exist nonsingular $n \times n$ matrices $H_{k}$ and $n \times n$ matrices $\mathscr{P}_{k}, \mathscr{2}_{k}$ satisfying (4.6), $k \in[m, n]$, such that

$$
X_{k}=S_{k}^{T} H_{k}, \quad U_{k}=C_{k}^{T} H_{k},
$$

where $\left(\begin{array}{l}S \\ C\end{array}\right)$ is a conjoined basis of (4.5) satisfying $C^{T} C-S^{T} S=I$ (or, equivalently, $C C^{T}$ $\left.S S^{T}=I, S C^{T}=C S^{T}\right)$. The matrices $\mathscr{P}$ and 2 are given by the formulas

$$
\begin{aligned}
& \mathscr{P}_{k}=H_{k+1}^{T-1}\left\{\left(\mathscr{C}_{k} X_{k}+\mathscr{D}_{k} U_{k}\right)^{T} U_{k}-\left(\mathscr{A}_{k} X_{k}+\mathscr{B}_{k} U_{k}\right)^{T} X_{k}\right\} H_{k}^{-1}, \\
& \mathscr{2}_{k}=H_{k+1}^{T-1}\left\{\left(\mathscr{A}_{k} X_{k}+\mathscr{B}_{k} U_{k}\right)^{T} U_{k}-\left(\mathscr{C}_{k} X_{k}+\mathscr{D}_{k} U_{k}\right)^{T} X_{k}\right\} H_{k}^{-1} .
\end{aligned}
$$

Proof. Let $H$ be any matrix satisfying $H^{T} H=U^{T} U-X^{T} X$, that is, $H=G D$, where $D$ is the (unique) symmetric positive definite matrix satisfying $D^{2}=U^{T} U-X^{T} X$ and $G$ is any orthogonal matrix. Denote $\mathscr{E}=(U+X) H^{-1}, \tilde{\mathscr{E}}=(U-X) H^{-1}$. Then the fact that $\left(\begin{array}{l}X \\ U\end{array}\right)$ is a conjoined basis implies that

$$
\begin{aligned}
\mathscr{L}_{k+1} & =\left(U_{k+1}+X_{k+1}\right) H_{k+1}^{-1} \\
& =\mathscr{L}_{k} H_{k}\left(U_{k}+X_{k}\right)^{-1}\left(U_{k}^{T}-X_{k}^{T}\right)^{-1}\left(U_{k}^{T}-X_{k}^{T}\right)\left(U_{k+1}+X_{k+1}\right) H_{k+1}^{-1} \\
& =\mathscr{L}_{k} H_{k}^{T-1}\left(U_{k}^{T}-X_{k}^{T}\right)\left(U_{k+1}+X_{k+1}\right) H_{k+1}^{-1} \\
& =\mathscr{L}_{k}\left(\mathscr{P}_{k}^{T}+\mathscr{Q}_{k}^{T}\right) .
\end{aligned}
$$

By the same computation, we get

$$
\tilde{\mathscr{E}}_{k+1}=\tilde{\mathscr{E}}_{k}\left(\mathscr{P}_{k}^{T}-\mathscr{2}_{k}^{T}\right) .
$$

Set

$$
S_{k}=\frac{1}{2}\left(\mathscr{E}_{k}^{T}-\tilde{\mathscr{E}}_{k}^{T}\right), \quad C_{k}=\frac{1}{2}\left(\mathscr{E}_{k}^{T}+\tilde{\mathscr{E}}_{k}^{T}\right) .
$$

Then we have

$$
\begin{aligned}
& S_{k+1}=\frac{1}{2}\left(\mathscr{L}_{k+1}^{T}-\tilde{\mathscr{E}}_{k+1}^{T}\right)=\frac{1}{2}\left[\left(\mathscr{P}_{k}+\mathscr{2}_{k}\right) \mathscr{L}_{k}^{T}-\left(\mathscr{P}_{k}-\mathscr{2}_{k}\right) \tilde{\mathscr{L}}_{k}^{T}\right]=\mathscr{P}_{k} S_{k}+\mathscr{2}_{k} C_{k}, \\
& C_{k+1}=\frac{1}{2}\left(\mathscr{L}_{k+1}^{T}+\tilde{\mathscr{E}}_{k+1}^{T}\right)=\frac{1}{2}\left[\left(\mathscr{P}_{k}+\mathscr{2}_{k}\right) \mathscr{L}_{k}^{T}+\left(\mathscr{P}_{k}-\mathscr{2}_{k}\right) \tilde{\mathscr{L}}_{k}^{T}\right]=\mathscr{2}_{k} S_{k}+\mathscr{P}_{k} C_{k} .
\end{aligned}
$$


Further,

$$
\begin{aligned}
C_{k} C_{k}^{T}-S_{k} S_{k}^{T} & =\frac{1}{4}\left(\mathscr{E}_{k}^{T}+\tilde{\mathscr{E}}_{k}^{T}\right)\left(\mathscr{L}_{k}+\tilde{\mathscr{E}}_{k}\right)-\frac{1}{4}\left(\mathscr{L}_{k}^{T}-\tilde{\mathscr{E}}_{k}^{T}\right)\left(\mathscr{E}_{k}-\tilde{\mathscr{E}}_{k}\right) \\
& =\frac{1}{2}\left(\mathscr{L}_{k}^{T} \tilde{\mathscr{L}}_{k}+\tilde{\mathscr{L}}_{k}^{T} \mathscr{E}_{k}\right) \\
& =\frac{1}{2} H_{k}^{T-1}\left[\left(U_{k}^{T}-X_{k}^{T}\right)\left(U_{k}+X_{k}\right)+\left(U_{k}^{T}+X_{k}^{T}\right)\left(U_{k}-X_{k}\right)\right] H_{k}^{-1} \\
& =\frac{1}{2} H_{k}^{T-1}\left(2 U_{k}^{T} U_{k}-2 X_{k}^{T} X_{k}\right) H_{k}^{-1}=I,
\end{aligned}
$$

and similarly $C_{k} S_{k}^{T}-S_{k} C_{k}^{T}=0$. The last two identities imply that the matrix ( $\left.\begin{array}{ll}C & S \\ S & C\end{array}\right)$ is symplectic. Hence, its transpose has the same property, which means that $\left(\begin{array}{l}S \\ C\end{array}\right)$ is a conjoined basis and $C^{T} C-S^{T} S=I$ holds. Finally, from the hyperbolic system (4.5) and the identities for its solution $\left(\begin{array}{l}S \\ C\end{array}\right)$, we have

$$
\mathscr{P}_{k}=C_{k+1} C_{k}^{T}-S_{k+1} S_{k}^{T}, \quad \mathscr{2}_{k}=S_{k+1} C_{k}^{T}-C_{k+1} S_{k}^{T}
$$

and by a direct computation, we get $\mathscr{P} \mathscr{P}^{T}-2^{2} \mathscr{2}^{T}=I$ and $\mathscr{P} \mathscr{2}^{T}=\mathscr{2} \mathscr{P} T$, which, by the same argument as above, implies that also $\mathscr{P}^{T} \mathscr{P}-\mathscr{2}^{T} \mathscr{Q}=I$ and $\mathscr{P}^{T} \mathscr{2}-\mathscr{2}^{T} \mathscr{P}=0$. This completes the proof.

Remark 4.2. Hyperbolic Prüfer transformation suggests an open problem in the transformation theory of (1.1), which can be explained as follows. In the hyperbolic Prüfer transformation, a conjoined basis $\left(\begin{array}{l}X \\ U\end{array}\right)$ is expressed in the form $(4.8)$, where $\left(\begin{array}{l}S \\ C\end{array}\right)$ is a conjoined basis of the hyperbolic system (4.5). By the classical Prüfer transformation for (1.1) (established in [7]), a conjoined basis of (1.1) is expressed by (4.8), but $\left(\begin{array}{l}S \\ C\end{array}\right)$ is a conjoined basis of the trigonometric SDS

$$
S_{k+1}=\mathscr{P}_{k} S_{k}+\mathscr{2}_{k} C_{k}, \quad C_{k+1}=-\mathscr{Q}_{k} S_{k}+\mathscr{P}_{k} C_{k}
$$

(similarly, as in the "hyperbolic" case, the terminology trigonometric system is justified by the fact that in the scalar case $n=1$, solutions of (4.16) can be expressed via classical trigonometric sine and cosine functions). Observe that hyperbolic and trigonometric systems are SDSs whose matrices satisfy (in addition to (1.2))

$$
\mathscr{S}^{T}\left(\begin{array}{ll}
I & 0 \\
0 & I
\end{array}\right) \mathscr{Y}=\left(\begin{array}{ll}
I & 0 \\
0 & I
\end{array}\right),
$$

respectively,

$$
\mathscr{S}^{T}\left(\begin{array}{cc}
I & 0 \\
0 & -I
\end{array}\right) \mathscr{S}=\left(\begin{array}{cc}
I & 0 \\
0 & -I
\end{array}\right) .
$$

Now, let $\mathcal{N}$ be any $2 n \times 2 n$ matrix and denote by $\mathscr{G}_{\mathcal{N}}$ the subgroup of $2 n \times 2 n$ symplectic matrices satisfying

$$
\mathscr{S}^{T} \mathcal{N} \mathscr{S}=\mathcal{N}
$$


The open problem is under what conditions on (1.1) any conjoined basis of this system can be expressed in the form (4.8) where $(\underset{C}{S})$ is a conjoined basis of the SDS (1.1) whose matrix $\mathscr{Y} \in \mathscr{G}_{\mathcal{N}}$.

\section{References}

[1] R. P. Agarwal, Difference Equations and Inequalities: Theory, Methods and Applications, 2nd ed., Monographs and Textbooks in Pure and Applied Mathematics, vol. 228, Marcel Dekker, New York, 2000.

[2] C. D. Ahlbrandt and A. C. Peterson, Discrete Hamiltonian Systems: Difference Equations, Continued Fractions, and Riccati Equations, Kluwer Texts in the Mathematical Sciences, vol. 16, Kluwer Academic Publishers, Dordrecht, 1996.

[3] M. Bohner, Linear Hamiltonian difference systems: disconjugacy and Jacobi-type conditions, J. Math. Anal. Appl. 199 (1996), no. 3, 804-826.

[4] M. Bohner and O. Došlý, Oscillation of symplectic dynamic systems on time scales, to appear in ANZIAM J.

[5] Disconjugacy and transformations for symplectic systems, Rocky Mountain J. Math. 27 (1997), no. 3, 707-743.

[6] , Trigonometric transformations of symplectic difference systems, J. Differential Equations 163 (2000), no. 1, 113-129.

[7] _ The discrete Prüfer transformation, Proc. Amer. Math. Soc. 129 (2001), no. 9, 27152726.

[8] S. Chen and L. Erbe, Oscillation results for second order scalar and matrix difference equations, Comput. Math. Appl. 28 (1994), no. 1-3, 55-69.

[9] O. Došlý and R. Hilscher, Linear Hamiltonian difference systems: transformations, recessive solutions, generalized reciprocity, Dynam. Systems Appl. 8 (1999), no. 3-4, 401-420.

[10] O. Došlý and Z. Pospíśil, Hyperbolic transformation and hyperbolic difference systems, Fasc. Math. 32 (2001), 25-48.

[11] S. N. Elaydi, An Introduction to Difference Equations, 2nd ed., Undergraduate Texts in Mathematics, Springer-Verlag, New York, 1999.

[12] L. Erbe and P. Yan, On the discrete Riccati equation and its applications to discrete Hamiltonian systems, Rocky Mountain J. Math. 25 (1995), no. 1, 167-178.

[13] P. Hartman, Difference equations: disconjugacy, principal solutions, Green's functions, complete monotonicity, Trans. Amer. Math. Soc. 246 (1978), 1-30.

[14] W. G. Kelley and A. Peterson, Difference Equations. An Introduction with Applications, Academic Press, Massachusetts, 1991.

[15] H. Prüfer, Neue herleitung der Sturm-Liouvilleschen Reihenentwicklung stetiger funktionen, Math. Ann. 95 (1926), 499-518 (German).

Ondřej Došlý: Department of Mathematics, Masaryk University, Janáčkovo nám. 2a, 66295 Brno, Czech Republic

E-mail address: dosly@math.muni.cz 


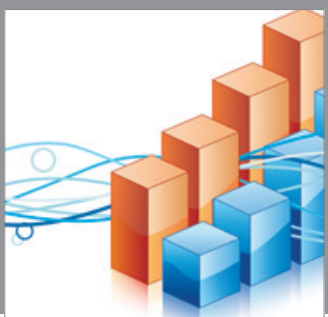

Advances in

Operations Research

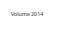

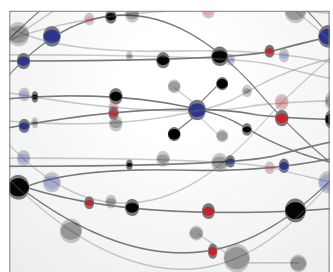

\section{The Scientific} World Journal
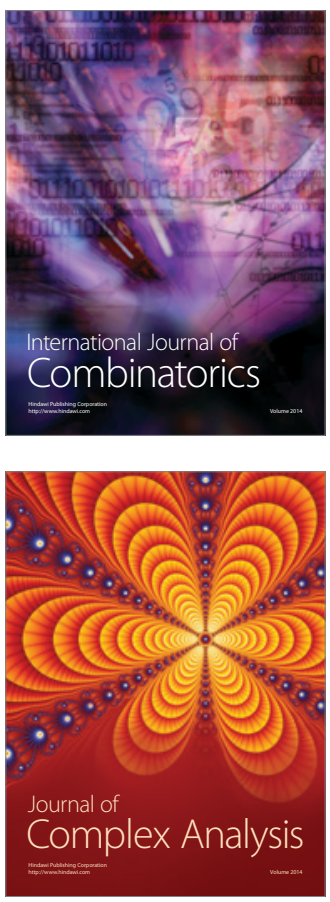

International Journal of

Mathematics and

Mathematical

Sciences
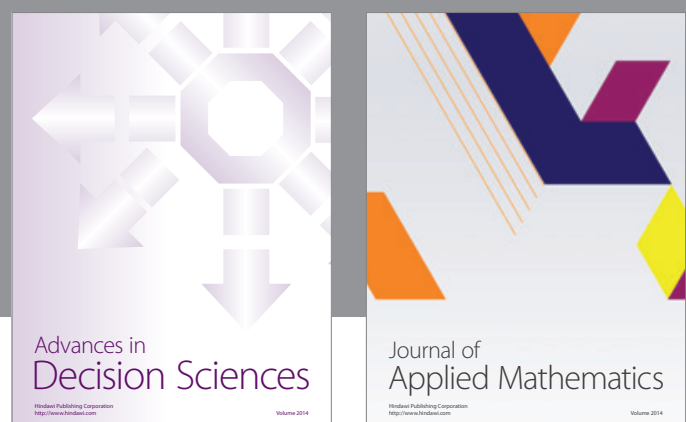

Journal of

Applied Mathematics
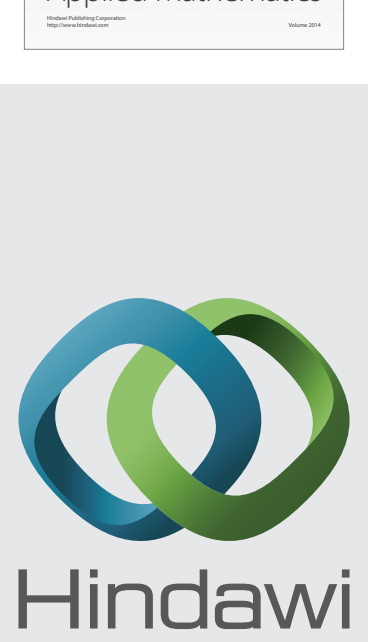

Submit your manuscripts at http://www.hindawi.com
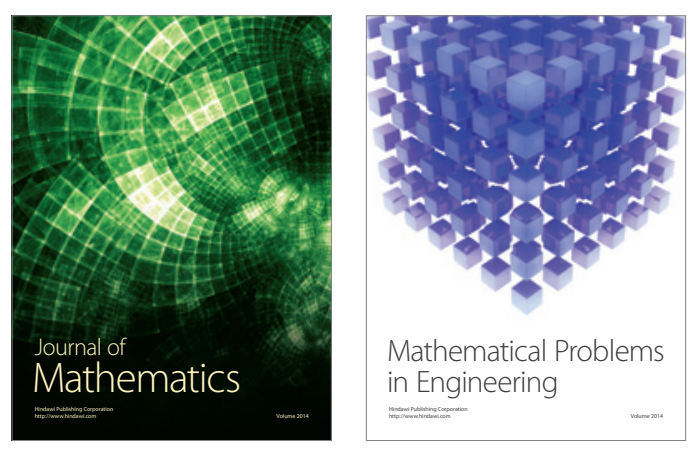

Mathematical Problems in Engineering
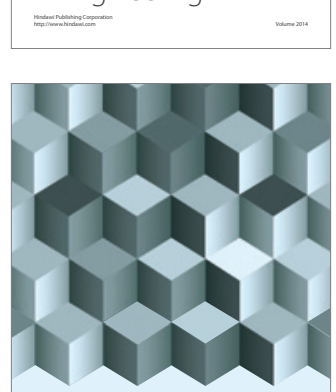

Journal of

Function Spaces
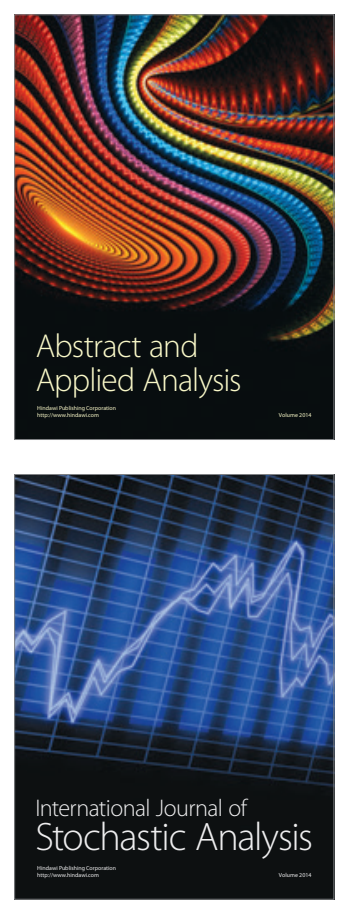

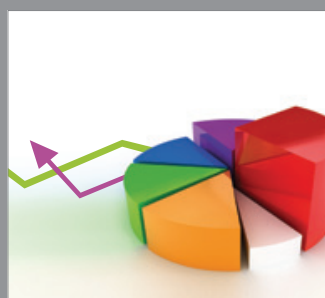

ournal of

Probability and Statistics

Promensencen
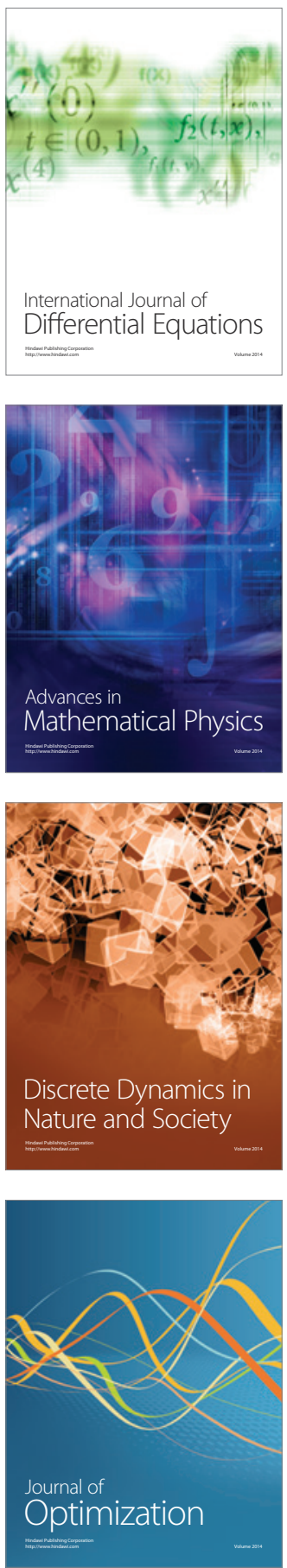